\title{
Navigation Aids Performance Evaluation for Precision Approaches
}

\author{
Pier Domenico Tromboni and Giovanni B. Palmerini \\ Università degli studi di Roma “La Sapienza”, via Eudossiana, 18-00184 Roma, Italy \\ Correspondence should be addressed to Pier Domenico Tromboni, pierdo.tromboni@libero.it
}

Received 24 June 2009; Revised 3 January 2010; Accepted 22 February 2010

Academic Editor: Dany Dionne

Copyright ( $) 2010$ P. D. Tromboni and G. B. Palmerini. This is an open access article distributed under the Creative Commons Attribution License, which permits unrestricted use, distribution, and reproduction in any medium, provided the original work is properly cited.

\begin{abstract}
The paper deals with the evaluation of the expected performance of aircraft approaches and landings operated with different navigation systems, both traditional and satellite-based. Flight dynamics characteristics and control authority of the approaching aircraft are considered in order to obtain an overall manoeuvre evaluation. The technique from the presented analysis applies to different operative conditions, taking into account aircraft requirements, navigation systems features, and environmental constraints. The aim is to offer a tool to be used in the very preliminary design phase for system performance analysis in different scenarios, such as airport ground systems adoption and air traffic control requirements compliance; later, the same tool can be tuned to complement and direct the required real flight trials to validate an already fielded solution. A numerical code referring to the presented analytical model has been implemented and some applications concerning the system's performance evaluation and planning are proposed to illustrate the algorithm capabilities. The tool and the proposed analysis technique indeed are successful in providing a quantitative assessment of the differences among several possible approaches.
\end{abstract}

\section{Introduction}

Air traffic is increasing and it is expected to intensify between 2008 and 2030 [1]. Medium-term forecast 2009-2015 [2] is that there will be 11.7 million IFR flight movements in the EUROCONTROL Statistical Reference Area (ESRA), 16\% more than 2008. Even with a decline by nearly 5\% in 2009, in the later years the growth recovers to more typical rates of $3.5 \%-4.5 \%$ per year and it is forecast to increase from 16.5 to 22.1 million IFR flight movements in 2030, with the rates of $2.3 \%-3.5 \%$ per year starting from 2015. These data justify reconsidering the actual aviation platform and all its supporting segments, such as airport capacity, ground and avionic equipments, flight procedures and of course navigation aids (navaids).

In the latter field, which is the subject of this paper, the main innovation is represented by satellite-based navigation, with the satellite technology deemed as mature enough to support Approach Procedure with Vertical guidance (APV) operations in terminal area and CAT II precision approach and landing starting from 2009 [3]. Traditional ground navigation aids infrastructure needs to be harmonised with incoming global navigation satellite systems in order to guarantee the use of satellite equipments as primary means in all the phases of the flight. From the air traffic control point of view, the increase in traffic capacity and the availability of the new technology involve a reassessment of the managed airspace, as well as a novel arrangement of the flight procedures.

These considerations especially apply to the terminal phase of the flight, which is the most critical and the bottleneck of the current system procedures. Either upgrades to existing systems to assist in landing or new installations are needed. Of course such investments require a detailed analysis to assess the benefit-to-cost ratio, and this analysis must be based on a technical evaluation of the different system performance, as well as on the specific selected site characteristics, like orography, aircraft class, airport infrastructure, navigation aids, and meteorological environment. The availability of software tools, which are able to help in this planning phase, indicating the expected performance, and/or highlighting possible deficiencies of existing or 


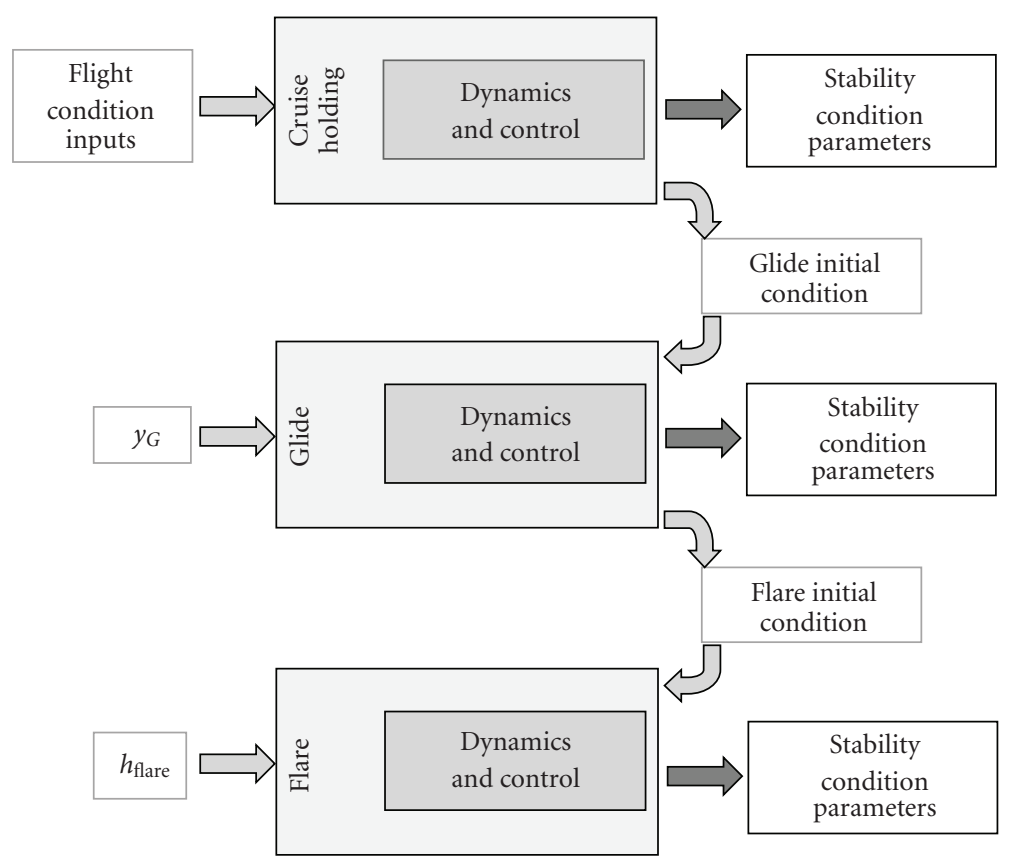

FIgURE 1: Data flow chart of the implemented flight phases.

designed infrastructures at different sites is therefore of interest. Later, once a navigation aid infrastructure has been actually installed, simulations can be useful again in the validation of the navaid behaviour with respect to the specific scenario and the other existing infrastructures in the area, and to identify the overall preferred solutions to exploit the instrumented environment. Obviously, these tools cannot substitute actual flight data, but should at least reduce the volume of data required to gain an understanding of the possible scenarios and of the differences among possible suitable solutions.

This paper aims to present a possible way to investigate the subject, including a number of issues that should be taken into account while designing this sort of tools. These issues have been integrated in a common structure, allowing for the analysis of the approach and landing phase. The proposed architecture has been implemented in a numerical code, and a set of actual flight data, gathered during flight tests performed at the Milano Linate airport (Italy) in December 2005, have been considered to validate the technique.

Presented material has been organized as follows: first of all some operative concepts regarding instrument approach are recalled in order to identify the environment investigated during the proposed tool applications. The following sections are devoted to the selection of the models to be used and to the flow of the algorithm to analyze the scenario; the process is specialized by including the values of the parameters appropriate for a realistic case and a specific aircraft. Then, the characteristics of the possible navigation aids which can be introduced in the scheme are depicted, followed by a short recall of the environmental quantities of interest. Finally, the results of the proposed application for a specific landing case are presented and discussed to validate the approach.

\section{The Phases of the Terminal Approach}

A general flow chart (Figure 1) reports the sequence of the flight conditions experienced by the aircraft during en-route, approach, and landing phase (with special reference to flare segment). Three different blocks can be easily identified:

(i) cruise: the flight phase immediately before the ILS signal acquisition,

(ii) glide: an approximately steady descent phase aiming at the runway, with a glide path to be produced by either the classical ILS facility or by the GBAS,

(iii) flare: the very last, nonlinear part of the landing trajectory exploited by the crew to align the aircraft to the runway.

The three blocks represent three different flight dynamics and control models, related to different portions of the flight sequence. It has to be explicitly remarked how the terms technically indicate the functional characteristics of each phase, and are not limited to their specific meaning in the air traffic control tradition. As an example, consider the glide, which refers to a vertical guidance of an aircraft descending along a linear path with a certain rate; it does not necessarily imply an approach phase segment, but it also applies to a variation of altitude commanded by the pilot during a cruise phase. Nevertheless, the approach application will be the focus in this paper because it better allows to compare the performance of different navaid systems.

Such a partition in modules has been driven by the need to describe each phase with different stability and control parameters, both for longitudinal and lateral flight dynamics: in fact, the blocks differ in the implemented algorithm because of the control models and of the state variable sets 
adopted in each phase of the flight. Parameters and variables describe

(i) dynamics and control model (i.e., state variables set definition),

(ii) flight condition (i.e., operative altitude and air speed),

(iii) aircraft typology (i.e., dimensions and mass distribution),

(iv) aircraft aerodynamic model (i.e., stability and control derivates).

A uniform flight condition was targeted in each phase to obtain the expected behaviour in a smooth flight. To this aim, the applied algorithms and the state and control variables adopted inside each block can differ.

Blocks are correlated by internal input-output relationship. The first block computes the steady state related to the imposed initial conditions, solves for the dynamics, and generates the output parameters for an assigned exit condition, then passes the kinematic state vector-including both translational (position and velocity) and rotational (attitude and angular rate) dynamics variables-at the end of each phase as the initial condition at the specific time for the following interval. The small blocks on the left recall the governing quantities during each phase, that is, the ideal glide path during the 3 degrees constant, line centered descent $\left(y_{G}\right)$, and the height of the regular flare is commanded in the final step $\left(h_{\text {flare }}\right)$.

A tool has been developed in MATLAB to simulate the approach, implementing different modules specifically tailored to each flight phase (the selection of the Matlab environment is due to its capability to handle matrices and easiness of use, as well as to the wide choice of available routines to help in control design).

\section{The Model and Its Implementation}

The intended analysis should be based on a model for the flight mechanics of the approaching/landing aircraft. The scheme proposed by Bryson for a controlled flight [4] has been selected, with an algorithm based on the linearized motion equations obtained by perturbing an equilibrium configuration typical of the descent phase. Resulting relations assume the classical aspect

$$
\begin{aligned}
& \dot{x}=F x+G u, \\
& y=H x+L u,
\end{aligned}
$$

where $F$ represents the natural dynamics of the state $x$ and $G$ is the distribution matrix for the control vector $u$. The controlled dynamics is completed by an observation section, which does provide the relation among the measured variables $y$ and the state and control vectors, as function of an observability matrix $\mathrm{H}$ and of a control/observability matrix $L$.

As customary in flight mechanics, the linearized model has been divided into two uncoupled channels, one relevant to the longitudinal dynamics and the other one to the lateraldirectional. Following Bryson, with classical notation also reported in the Appendix and related Figure 9, the state vector for the longitudinal motion is (with $\delta$ indicating the small perturbation around the equilibrium condition)

$$
x=\left[\begin{array}{lllll}
\delta u & \delta w & q & \delta \vartheta & h
\end{array}\right]^{T},
$$

the control vector is represented by $u=\left[\begin{array}{ll}\delta(\delta e) & \delta(\delta t)\end{array}\right]^{T}$, and the observed variables are $y=\left[\begin{array}{ll}\delta u & \dot{h}\end{array}\right]^{T}$.

Similarly, the lateral-directional motion has been defined by the following state vector:

$$
x=\left[\begin{array}{llllll}
\delta v & \delta r & \delta p & \delta \varphi & \delta \psi & y_{\mathrm{CL}}
\end{array}\right]^{T},
$$

the control is $u=\left[\begin{array}{ll}\delta a & \delta \rho\end{array}\right]^{T}$ and the measurements are $y=\left[\begin{array}{lll}\delta v & a_{y} & y_{\mathrm{CL}}\end{array}\right]^{T}$. The matrices $F$ and $G$ (which take into account the aerodynamic derivatives) have been reported in the appendix for both the cases.

To provide a realistic case, the aircraft will be considered as equipped with a Stability Augmentation System (SAS), and therefore targeting steady conditions in the different phases for both longitudinal and lateral control. These actions are computed via a Linear Quadratic Regulator (LQR), which certainly applies to the selected linearized dynamics and is capable to provide the optimal solution by minimizing a quadratic performance index:

$$
J=\frac{1}{2} \int_{0}^{\infty}\left(x^{T} A x+u^{T} B u\right) \cdot d t
$$

with $A$ and $B$ weighting matrices associated with the tolerances accepted on the state and with the cost of the control action.

In order to represent the real behaviour, a noisy (i.e., with a nondeterministic component) output for the sensors providing the measurements has to be considered in the observation equation $\left(B_{w}\right)$. At the same time a process noise on the dynamics ( $w$ given by wind, or errors on the $F$ matrix) has to be included to take into account unmodeled and/or quantitatively unforeseeable effects. Indeed, the knowledge of the state evolution, required to compute the performance index, is intrinsically based on an estimation function. The optimal estimate, meaning that it does provide the minimum sum of the variances for each state component under the generally accepted hypothesis of white noise disturbances, is given by the Kalman filter [5-7]. In the overall control scheme (see Figure 2), such an estimate is provided to a regulator, that closes the loop, feeding back the control action required to follow the dynamics identified as optimal by means of the previous minimization criteria. Bryson [4] discusses the close similarity among the solutions of the optimal regulation and of the optimal estimation problems. As a result, the complete solution scheme can be recast as a Linear Quadratic Gaussian (LQG) compensator.

3.1. An Example of Model Implementation. The previous model has been applied to the Cessna Citation S550 aircraft. 


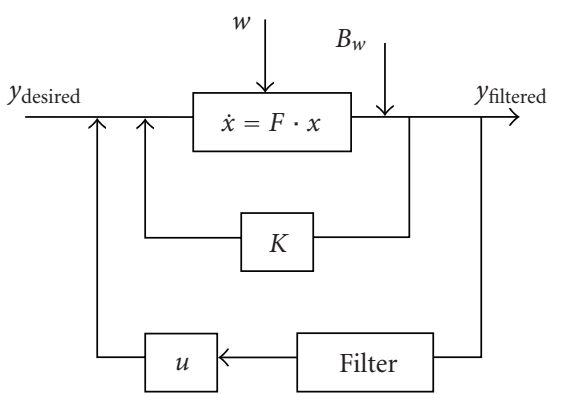

Figure 2: Schematic flow chart of the implemented dynamic system.

The type has been chosen for the opportunity to perform a comparison with available experimental data, gathered during a trial campaign performed at the Milano Linate airport and aimed to a GBAS equipment test validation ${ }^{1}$. In such a frame, all longitudinal and lateral-directional stability and control derivates of the Cessna Citation S550 have been ad hoc evaluated starting from basic aircraft data (i.e., reference geometry, flight condition, and mass data), in order to be later used to compare the code behaviour with real data from an instrumental glide phase approach in Milano. For the longitudinal and lateral-directional dynamics, the state and control matrices assume the form showed in Appendix, computed according to Nelson [8].

The effects of the weighting matrices $A$ and $B$ on the control output have been evaluated by means of an iterative and exhaustive search aimed to obtain a behaviour comparable to real data. The initial guess for these numerical trials has been selected according to Bryson [4], where the authority of the different actuators has been assessed for typical cases. Then, as recalled later during the discussion of the results, the entries have been modified to have the simulations providing similar excursions in interesting kinematic parameters as the ones observed, and therefore deemed as acceptable, in real tests. As a result, the weighting matrices $A$ and $B$, associated with the tolerances on the state and with the cost of the control action, respectively, assume for the Citation S550 simulation the final form showed in the appendix.

\section{Errors Budget for Navaids}

Different satellite-based systems have been considered as possible sensors: GPS stand-alone, ground-based augmented system (GBAS), and space-based augmented system (SBAS). Even if availability, continuity, and integrity considerations have been taken into account, the study has been focused on the accuracy which has been chosen as the comparison parameter among different systems. Several experimental campaigns have been purposely set-up $[9,10]$ to characterize accuracy figures. Both vertical and horizontal accuracy errors have been computed and included as sensors' noises in the lateral and longitudinal motion equations implemented in the algorithm. In order to easily compare the total accuracy performance (i.e., 3D figures) of each considered navaid system, an overall performance parameter, labelled global accuracy error, has been introduced. Such a parameter is
TABLE 1: Sensor global accuracy error budget expressed in terms of average $\bar{\varepsilon}$ and variance $\bar{\sigma}^{2}$.

\begin{tabular}{|c|c|}
\hline \multicolumn{2}{|c|}{ ILS error } \\
\hline Average $[\mathrm{m}]$ & Variance* $\left[\mathrm{m}^{2}\right]$ \\
\hline 7.70 & 93.17 \\
\hline \multicolumn{2}{|c|}{ GPS error } \\
\hline Average $[\mathrm{m}]$ & Variance $\left[\mathrm{m}^{2}\right]$ \\
\hline 6.00 & 8.98 \\
\hline \multicolumn{2}{|c|}{ SBAS error } \\
\hline Average [m] & Variance $\left[\mathrm{m}^{2}\right]$ \\
\hline 3.00 & 1.75 \\
\hline \multicolumn{2}{|c|}{ GBAS error } \\
\hline Average [m] & Variance $\left[\mathrm{m}^{2}\right]$ \\
\hline 1.03 & 0.46 \\
\hline
\end{tabular}

* The high value of the variance can be noted. In the considered case, the calculated errors show a horizontal component $\left(\bar{\varepsilon}_{h}=5.83, \bar{\sigma}_{h}^{2}=25.80\right)$, better than the vertical one $\left(\bar{\varepsilon}_{v}=5.03, \bar{\sigma}_{v}^{2}=89.53\right)$.

expressed in terms of average $\bar{\varepsilon}_{\mathrm{GAE}}$ and variance $\bar{\sigma}_{\mathrm{GAE}}^{2}$ of the position expressed in geographical coordinates (i.e., latitude, longitude, and altitude) computed using the data collected in the appropriate time intervals:

$$
\begin{gathered}
\bar{\varepsilon}_{\mathrm{GAE}}=\sqrt{\left(\bar{\varepsilon}_{\mathrm{LAT}}\right)^{2}+\left(\bar{\varepsilon}_{\mathrm{LONG}}\right)^{2}+\left(\bar{\varepsilon}_{\mathrm{ALT}}\right)^{2}}, \\
\bar{\sigma}_{\mathrm{GAE}}^{2}=\sqrt{\left(\sigma_{\mathrm{LAT}}^{2}\right)^{2}+\left(\sigma_{\mathrm{LONG}}^{2}\right)^{2}+\left(\sigma_{\mathrm{ALT}}^{2}\right)^{2}} .
\end{gathered}
$$

Accuracy bounds offer the chance to "draw" a cylinder, whose radius is given by the accuracy parameter, surrounding the nominal flight path. This approach can be followed for either conventional (i.e., Instrumental Landing System (ILS)) and satellite-based (i.e., Global Positioning System (GPS), Ground Based Augmentation System (GBAS), Space Based Augmentation System (SBAS)) navaids, with the remark that for ILS, a cone, instead of a cylinder, will be used, as the ILS error is given by an angle and the corresponding position error increases proportionally to the distance from localizer (LOC) and Glide Slope (GS) antennas.

The adoption of the global accuracy error, as the analysis parameter, allows for an easy extension of the accuracy performance comparison to the ILS, whose experimental data are generally reported in terms of a so-called Path Definition Error (PDE), that is, the deviation between the desired flight path and the path flown by the aircraft. In fact, data gathered during flight tests show the actual flight path, affected by both horizontal and vertical accuracy errors due to the radiated localizer and glide slope signals, therefore providing the difference with respect to the nominal ILS path.

Figures adopted in simulations (and reported in following Table 1) have been obtained from different sources. In detail, ILS and GBAS data result from postprocessing of data collected during several flight trials. Regarding the ILS, real data of the terminal part of the approach part (i.e., beginning at 4 nautical miles-NM-before threshold) have been considered, and later extended at larger distances by means of an extrapolation to be used in following computation. 
TABLE 2: Velocity average and standard deviation of the wind.

\begin{tabular}{lccccc}
\hline & \multicolumn{2}{c}{ Wind velocity } & \multicolumn{2}{c}{ Wind direction } \\
Average $[\mathrm{m} / \mathrm{sec}]$ & St. Dev. $[\mathrm{m} / \mathrm{sec}]$ & Max $[\mathrm{m} / \mathrm{sec}]$ & Min $[\mathrm{m} / \mathrm{sec}]$ & Average [degree] & St. Dev. [degree] \\
\hline 12.18 & 6.49 & 25.09 & 0.63 & 272.24 & 66.28 \\
\hline
\end{tabular}

GPS and SBAS accuracies have been estimated referring to the literature $[11,12]$ and confirmed via ad hoc experiments with a Septentrio Polar RX2 receiver.

\section{Environment Characterization}

To simulate the perturbed dynamics, the wind has been modelled as external disturbance, describing its velocity as intensity and direction. The stochastic contribution to be introduced in the Kalman scheme has been evaluated from real data collected at the Milano-Linate site. Two sets of experimental data gathered on the same airport and at the same period have been considered, with the first one composed by ground measurements and the second one referred to an altitude profile. Average and standard deviations for the horizontal and vertical components have been accordingly evaluated. A similar approach has been used to estimate the direction of the prevailing wind, in order to include a meaningful range of possible wind headings in the simulation, and to provide correct components once the wind is projected along body frame axes $\left(u_{w}, v_{w}, w_{w}\right)$.

Table 2 reports the values resulting from an extensive analysis of the conditions observed at the airport site where flight tests have been carried out (Milano Linate, December 2005).

\section{Proposed Applications}

6.1. Instrument Approach. Approaches are classified as either precision or nonprecision, depending on the accuracy and capabilities of the navigational aids used. Precision approaches aim to provide lateral and vertical guidance to an aircraft pilot for landing, until the missed approach point is reached. Nonprecision approaches provide lateral course information only until reaching the MDA/H (Minimum Decision Altitude/Height), or beyond.

Precision approaches are permitted by means of appropriate ground systems as ILS, and microwave landing system (MLS). Also satellite-based systems can be used, such as GPS with vertical navigation via augmented information supplied by local (LAAS) or wide area (WAAS) systems, satellite-based (SBAS) or ground-based (GBAS).

Nonprecision approaches are aided by VHF Omnidirectional Range (VOR), Non-Directional Beacon (NDB) with complementary Automatic Direction Finder (ADF) installed on board, GPS (only used as secondary means under certain flight level), Tactical Air Navigation (TACAN) and Localizer.

Instrumental approaches generally involve different phases of flight. From the point of view of a pilot the most critical phases are arrival and approach (see Figure 3), further divided by ICAO [13] into three segments (initial, intermediate, and final).
While Arrival is defined as the phase where the pilot navigates to the Initial Approach Fix (IAF, a navaid or reporting point), and where holding can take place, the three approach segments can be shortly recalled as follows:

(i) initial approach segment: that segment of an instrument approach procedure between the initial approach fix and the intermediate approach fix or the final approach fix or point,

(ii) intermediate approach segment: that segment of an instrument approach procedure between either the intermediate approach fix and the final approach fix or point.

(iii) final approach: that segment between 12 and $4 \mathrm{NM}$ of straight flight descending at a set rate (usually an angle of between 2.5 and 6 degrees).

When aircraft is under radar control, air traffic controllers may replace some or all of these phases of the approach with radar vectoring, providing the headings on which the pilot should navigate his aircraft to the final approach, generally until the final approach aid coverage, for example, the ILS, which is then used for the final approach.

6.2. Approach Simulation. The proposed technique has been applied to the simulation of a GBAS alike approach of a Cessna Citation aircraft, with special focus on the flare phase. A GBAS system error, evaluated by means of the postprocessing of the flight test results, has been assumed. As far as it concerns the wind, the only stochastic component, with a deviation standard equal to the value previously given in Table 2, has been considered. In such a way, all the noises, both on the measurements and on the process, have been directly derived from real data.

The control parameters have been set-up by means of a validation process based on a recursive method, comparing numerical and experimental positioning errors until an appropriate match has been achieved. In detail, the aim was not to exactly reproduce the GBAS experimental trajectory, as such an attempt should have taken into account the evaluation of pilot's commands along that specific flight, losing the proposed analysis appeal for general cases. Instead the quest has been to stabilize the error around the null value, assuming extreme excursions similar to the ones obtained in the considered real flight trial. The magnitude of the control action, that is, the authority of the control, which enters into the index of (4) by means of the matrices $A$ and $B$, has been accordingly assessed. This approach differs from the one typical in "control" studies and is by far easier to develop, as it does not necessarily require difficult-toretrieve data as the time history of the pilot's commanded action. The instantaneous correction force supplied by the 


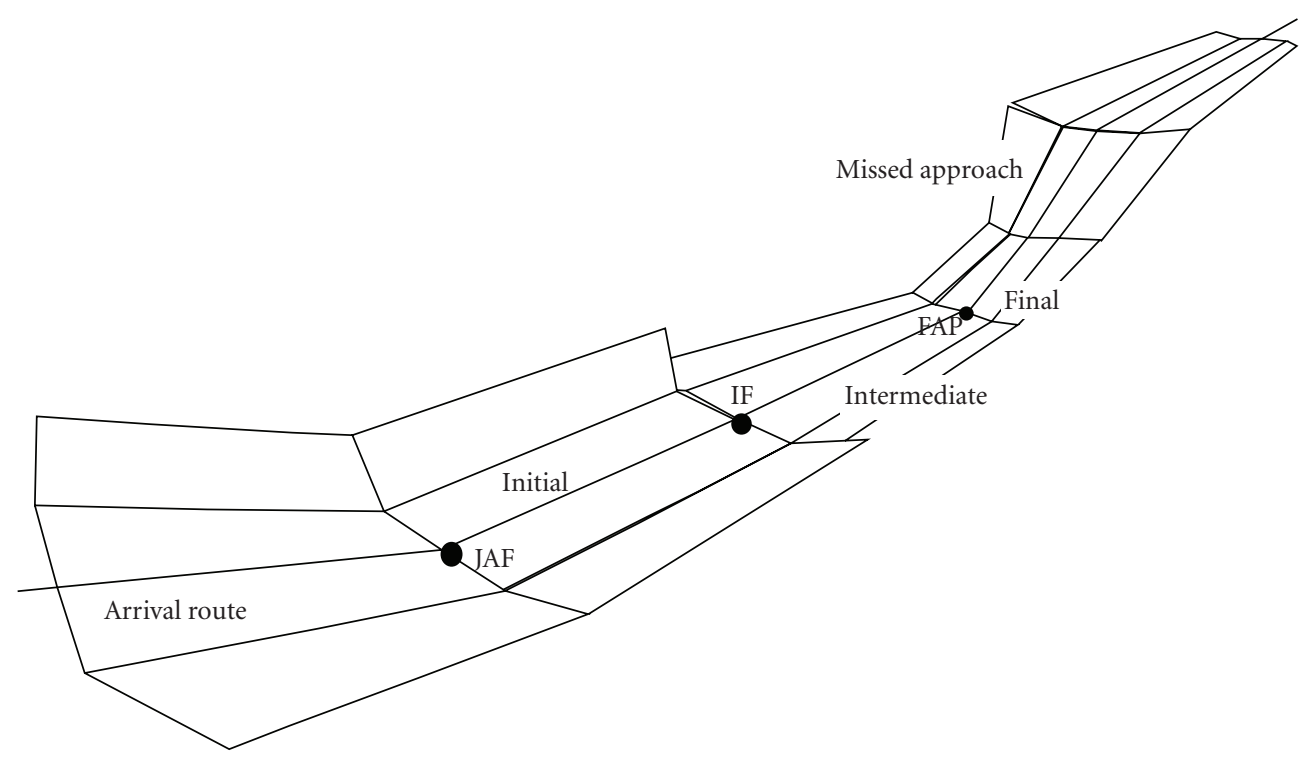

FIGURE 3: Instrumental approach segments [13].

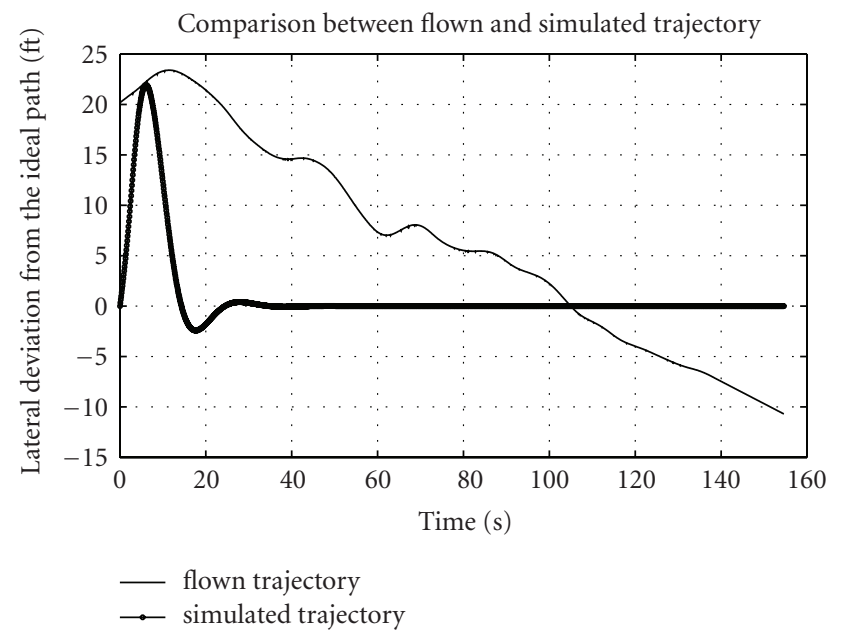

FIGURE 4: Numerical and experimental positioning error in a GBAS approach.

aircraft actuator has been kept unknown (if not specifically required) in the present case, and the focus remains on the kinematic variables, which are more interesting to investigate the navigation performance.

Figure 4 therefore reports the time history of the positioning error affecting the estimation of a trajectory, expressed in terms of lateral deviation from the ideal path. The experimental trajectory results from the postprocessing of data gathered during a GBAS flight trial session. The numerical one is the output of the simulation performed by using values typical of the experimental operative condition for both internal (i.e., sensor noise) and external (i.e., wind) perturbations. Focusing on the maximum shift from ideal path, a coherent behaviour of the simulated output can be seen, indicating that the selected control gains are appropriate to handle similar excursions.
The findings for the longitudinal channel simulation of a Cessna Citation are shown in Figure 5, referring to longitudinal state and control parameters time histories.

With the implemented control algorithm, the represented variables' values (i.e., horizontal speed, descent rate, and pitch angle of the pitch control surface) need almost 10 seconds to reach the steady state given by the imposed values for the state parameters (i.e., $\delta u=0 \mathrm{~m} / \mathrm{s} ; \vartheta=-10$ centirad) during the glide. The corresponding control to obtain such a stabilization is reported in terms of the variations of the elevator angle $(\delta e)$ and of the thrust $(\delta t)$. Simulation results match reasonable real world behaviour, also with respect to the time requested to stabilize the aircraft.

The validation of the code can be completed by the analysis of Figure 6, where the altitude profile obtained by the LQG algorithm (circle marked line) is shown to follow quite closely the ideal exponential path (solid line) during a flare. Again, noises applied (sensors and wind) match real world characteristics obtained by data sheets and on site measurements as previously indicated.

All the presented cases refer to the analysis of the performance of a specific aircraft while landing at a defined site, and using a selected, or a set of selected navigation aids. In a more general perspective, this approach can save on the number of specific flights devoted to this goal, by directing them to the more critical situations.

6.3. Comparison among Different Systems and Infrastructure Planning. A different point of view deals with planning issues. By using different modules to simulate several navigation satellite systems as sensors (i.e., GPS stand-alone, GBAS, SBAS), specifically implemented in the code, a performance comparison and an airspace management planning can be exploited. To illustrate this second possible application of the numerical code, a simulation of a glide approach has been performed for each proposed navigation satellite-based 


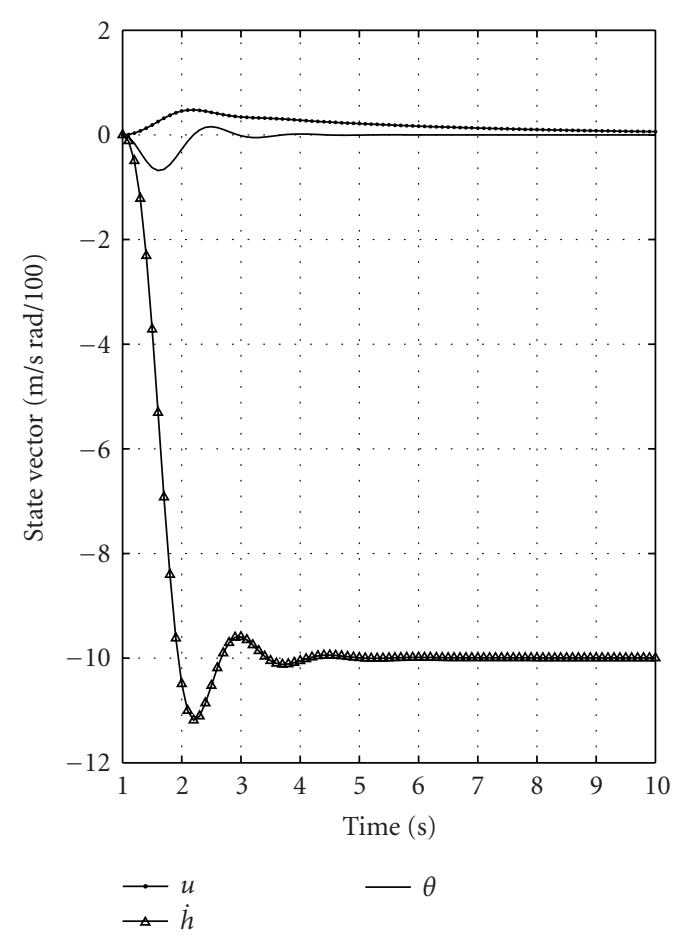

(a)

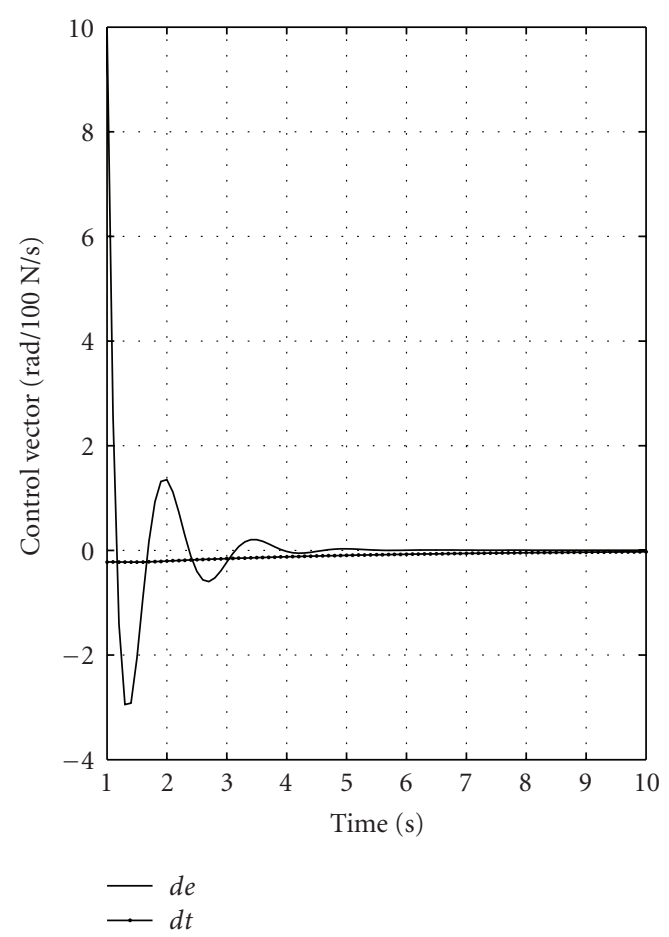

(b)

Figure 5: Time history of the kinematic variables (a) and of the control vector components (b) during a Cessna Citation simulated descent.

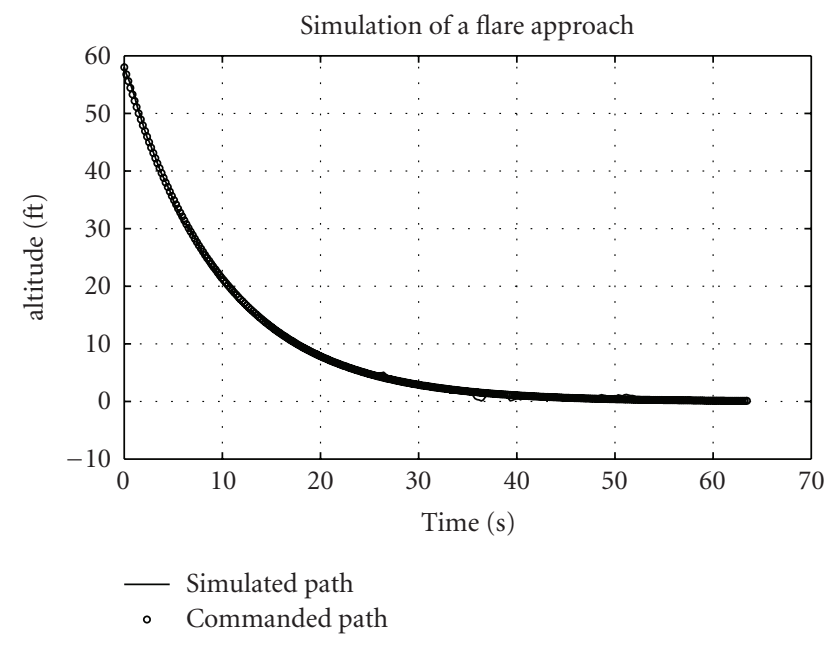

FIGURE 6: Longitudinal dynamics—-feed-back with Kalman filter.

system assuming the very same initial conditions. The same value of the external disturbance (i.e., wind), estimated under conservative hypothesis, has been assumed as well. The deviation in position from an ideal glide path has been chosen as a comparison parameter. In order to show the improvement in accuracy performance obtainable with satellite-based navigation systems, a traditional instrumental landing system (ILS) has been also considered.

By including as sensor noise the relevant numbers of the navaids under investigation for traditional ILS, GPS standalone, GPS aided by GBAS, high precision GPS based on the phase of the received signal and on-the-fly (or Real Time Kinematic (RTK)) solution of the ambiguity problem [14, $15]$, it is possible to have a complete scenario of the possible techniques for an instrumental landing. From the implementation point of view, the systems considered could require a different set of state variables to drive the simulation: every choice does not change the behaviour of the system, but enables instead a better comprehension of the influence of different navigation aids on the overall performance.

The longitudinal and lateral positioning errors for the proposed systems are shown in Figures 7 and 8, respectively. ILS trajectory, recovered from data recorded during the geometrical flare of a Cessna Citation aircraft, has been reconstructed as regression curve from experimental data [9]. Moreover, the same data set allows for an extrapolation concerning the farthest portion of the approach slope.

The oscillation of the instantaneous aircraft position around the average trajectory can be easily spotted, especially in lateral dynamics. This behaviour can be explained focusing on the fact that the adopted control parameters in LQG (i.e., the values for the entries in $A$ and $B$ matrices of the cost function $J$ ) have not been optimized for each considered navigation sensor but that a common value has been imposed instead. This choice should not be considered as a limit as the open algorithm could be easily modified and customized to specific control parameters.

In particular, Figure 7 shows the longitudinal deviation from the ideal path (circle marked line) obtained with an aircraft approaching with the considered, different sensors. Results show that GBAS system (dotted line) permits the best accuracy performance, while ILS makes the worst. At the 


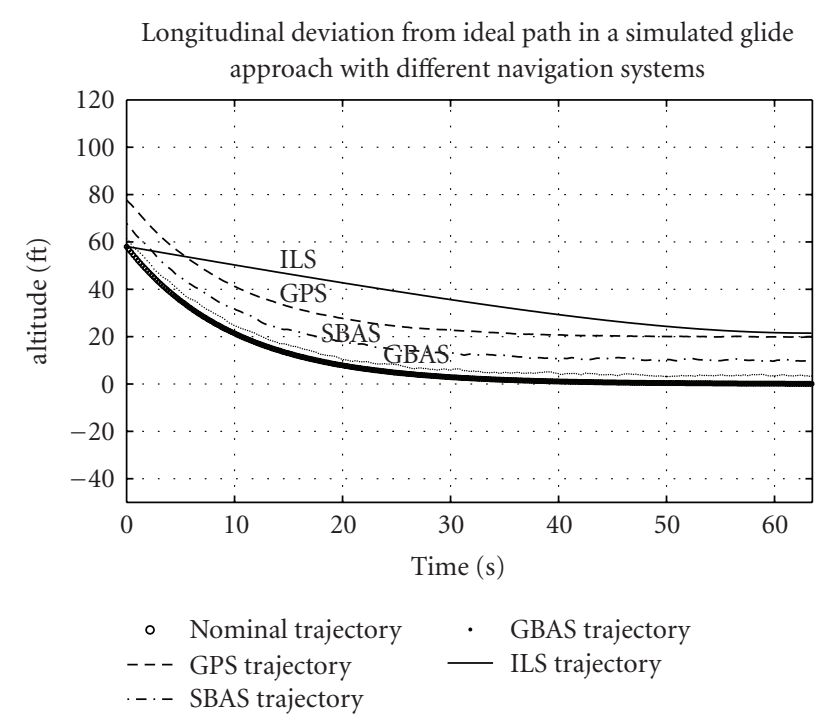

FIGURE 7: Longitudinal positioning comparison among different navigation systems.

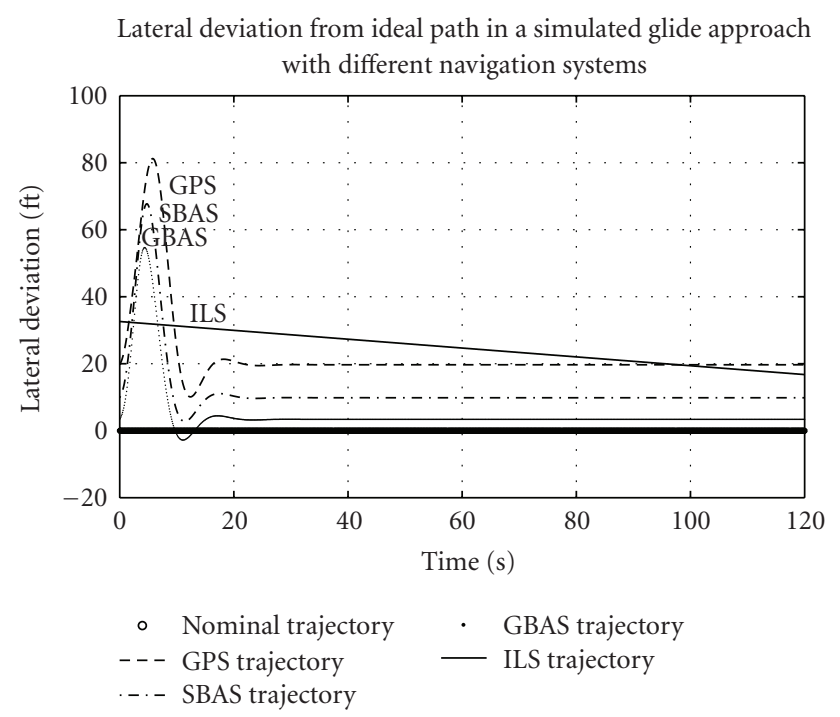

FIGURE 8: Lateral positioning comparison among different navigation systems.

final approach (around 3.8 NM from the runway threshold corresponding to $60 \mathrm{~s}$ in Figure 7), the lateral deviation passes from almost 4 feet for GBAS to more than 20 feet for ILS. A similar dynamic behaviour can be noticed for each sensor maintaining a different accuracy performance with respect to the nominal trajectory along the entire path.

Coherent results have been obtained by simulating (Figure 8) the lateral control of the same aircraft under the identical flight conditions and control settings. The use of a GBAS sensor permits to achieve a lateral error, that is, the distance between the ideal and the actually flown paths, better than an ILS approach ever since 5,7 NM from the runway threshold (corresponding to $7.9 \mathrm{~s}$ in Figure 8). On

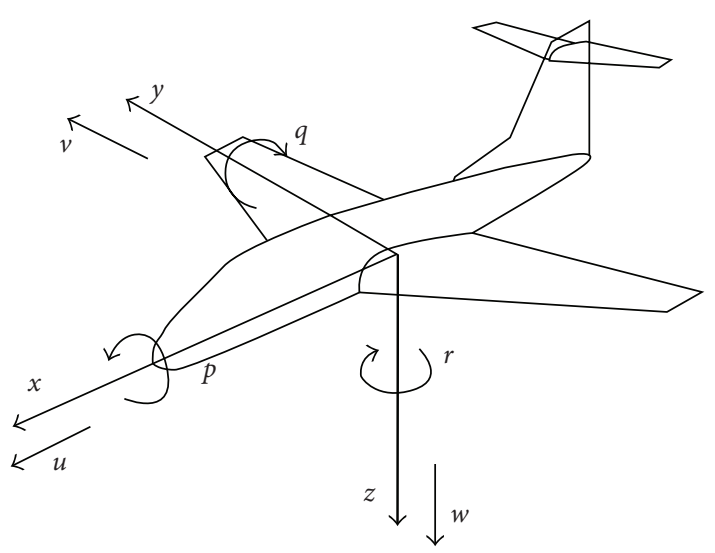

Figure 9: Adopted reference coordinate system.

the other side, ILS, if compared to a GPS stand-alone, allows for a more accuracy at distances larger than 2.2 NM from the threshold (i.e., 97.9 seconds in Figure 8).

Figures 7 and 8 clearly show how every considered navaid is able to fulfill CAT I requirements (associated RNP 0.02/40 as per ICAO [16]). Augmented GPS, that is, SBAS and GBAS, are also able to satisfy CAT II requirements (i.e., 0.01/15 associated RNP [16]).

\section{Conclusions}

A technique to preliminary evaluate navigation systems performance in terminal phase has been presented. In the frame of the classical linear control theory, the proposed approach allows to include site characteristics, typical noise components, and even partial-real flight data, providing a consistent estimate of the performance achievable with a defined navigation aid. At the same time, still building on a possibly incomplete set of real measurements, this approach can be used to compare different navigation systems performance. The numerical tool which implements the approach can be easily specialized to different sites and aircrafts, as to obtain a flexible planning tool to evaluate different options about navaid architectures for a specific airport. The approach has been exploited to obtain performance estimates for stand-alone, satellite-based augmented, and ground-based augmented GPS. Results for satellite-based navigation systems have been compared to traditional ILS performance.

The sets of sensors' and wind's data collected during real flight trials and used as inputs and comparison terms in the simulations validated the approach; they also offer an intrinsic valuable indication for further work.

Results confirmed that all the proposed sensors are capable of CAT I operations in the considered specific conditions, while CAT II is attainable with GBAS.

\section{Appendix}

With respect to (2) and (3) and the frame reported in Figure 9, the following relations hold. 
Longitudinal motion:

$$
F=\left[\begin{array}{ccccc}
\bar{X}_{u} & \bar{X}_{w} & -u_{0} \sin \vartheta_{0} & -g \cos \vartheta_{0} & 0 \\
Z_{u} & Z_{w} & u_{0} \cos \vartheta_{0} & -g \sin \vartheta_{0} & 0 \\
\bar{M}_{u} & \bar{M}_{w} & \bar{M}_{q} & 0 & 0 \\
0 & 0 & 1 & 0 & 0 \\
0 & -1 & 0 & u_{0} & 0
\end{array}\right],
$$

with

$$
\begin{aligned}
\bar{M}_{i} & =\frac{M_{i}+M_{\dot{w}} Z_{i}}{I_{y}}, \\
\bar{X}_{i} & =\frac{X_{i}+T_{i}}{m}, \\
G & =\left[\begin{array}{cc}
X_{\delta e} & T_{\delta t} \cos \varepsilon \\
Z_{\delta e} & -T_{\delta t} \sin \varepsilon \\
M_{\delta e} & 0 \\
0 & 0 \\
0 & 0
\end{array}\right] .
\end{aligned}
$$

Lateral motion:

$$
\begin{gathered}
F=\left[\begin{array}{cccccc}
Y_{v} & -u_{0} \cos \vartheta_{0} & u_{0} \sin \vartheta_{0} & g \cos \vartheta_{0} & 0 & 0 \\
\bar{N}_{v} & \bar{N}_{r} & \bar{N}_{p} & 0 & 0 & 0 \\
\bar{L}_{v} & \bar{L}_{r} & \bar{L}_{p} & 0 & 0 & 0 \\
0 & \tan \vartheta_{0} & 1 & 0 & 0 & 0 \\
0 & 1 & 0 & 0 & 0 & 0 \\
1 & 0 & 0 & 0 & V & 0
\end{array}\right], \\
G=\left[\begin{array}{cccc}
Y_{\delta a} & Y_{\delta \rho} \\
\bar{N}_{\delta a} & \bar{N}_{\delta \rho} \\
\bar{L}_{\delta a} & \bar{L}_{\delta \rho} \\
0 & 0 \\
0 & 0 \\
0 & 0
\end{array}\right] .
\end{gathered}
$$

Longitudinal state and control matrices for the Cessna Citation descent case:

$$
F=\left[\begin{array}{ccccc}
-0.062 & 0.186 & 0 & -0.299 & 0 \\
-0.378 & -0.766 & 1.579 & 0 & 0 \\
-0.025 & -10.99 & -1.067 & 0 & 0 \\
0 & 0 & 1 & 0 & 0 \\
0 & -1 & 0 & 1.7 & 0
\end{array}\right] \text {, }
$$

$$
G=\left[\begin{array}{cc}
0 & 1 \\
9.21 & 0 \\
-2.73 & 0 \\
0 & 0
\end{array}\right], \quad H=\left[\begin{array}{lllll}
1 & 0 & 0 & 0 & 0 \\
0 & 0 & 0 & 0 & 1
\end{array}\right], \quad L=\left[\begin{array}{ll}
0 & 0 \\
0 & 0
\end{array}\right]
$$

Lateral-directional state and control matrices for the Cessna Citation descent case:

$$
F=\left[\begin{array}{cccccc}
-0.106 & -1.579 & 0.031 & 0.299 & 0 & 0 \\
1.71 & -0.492 & -0.246 & 0 & 0 & 0 \\
-2.265 & 1.428 & -1.238 & 0 & 0 & 0 \\
0 & 0 & 1 & 0 & 0 & 0 \\
0 & 1 & 0 & 0 & 0 & 0 \\
1 & 0 & 0 & 0 & 1.7 & 0
\end{array}\right],
$$$$
G=\left[\begin{array}{cc}
0 & 0.092 \\
-0.616 & -1.379 \\
3.082 & 0.289 \\
0 & 0 \\
0 & 0 \\
0 & 0
\end{array}\right]
$$

$$
H=\left[\begin{array}{cccccc}
-0.106 & 0 & 0 & 0 & 0 & 0 \\
0 & 0 & 0 & 0 & 0 & 1
\end{array}\right], \quad L=\left[\begin{array}{cc}
0 & 0.092 \\
0 & 0
\end{array}\right]
$$

Weighting matrices $A$ and $B$ for the longitudinal dynamic for the Cessna Citation descent case:

$$
\begin{aligned}
& A=\left[\begin{array}{ccc}
30.8 & 0 & 0 \\
0 & 0.94 & 0 \\
0 & 0 & 40.8
\end{array}\right], \\
& B=\left[\begin{array}{cc}
4.59 & 0 \\
0 & 7.30
\end{array}\right] .
\end{aligned}
$$

Weighting matrices $A$ and $B$ for the lateral-directional dynamic for the Cessna Citation descent case:

$$
\begin{aligned}
& A=\left[\begin{array}{cc}
4 & 0 \\
0 & 0.04
\end{array}\right], \\
& B=\left[\begin{array}{ll}
1 & 0 \\
0 & 1
\end{array}\right] .
\end{aligned}
$$




\section{Notation}

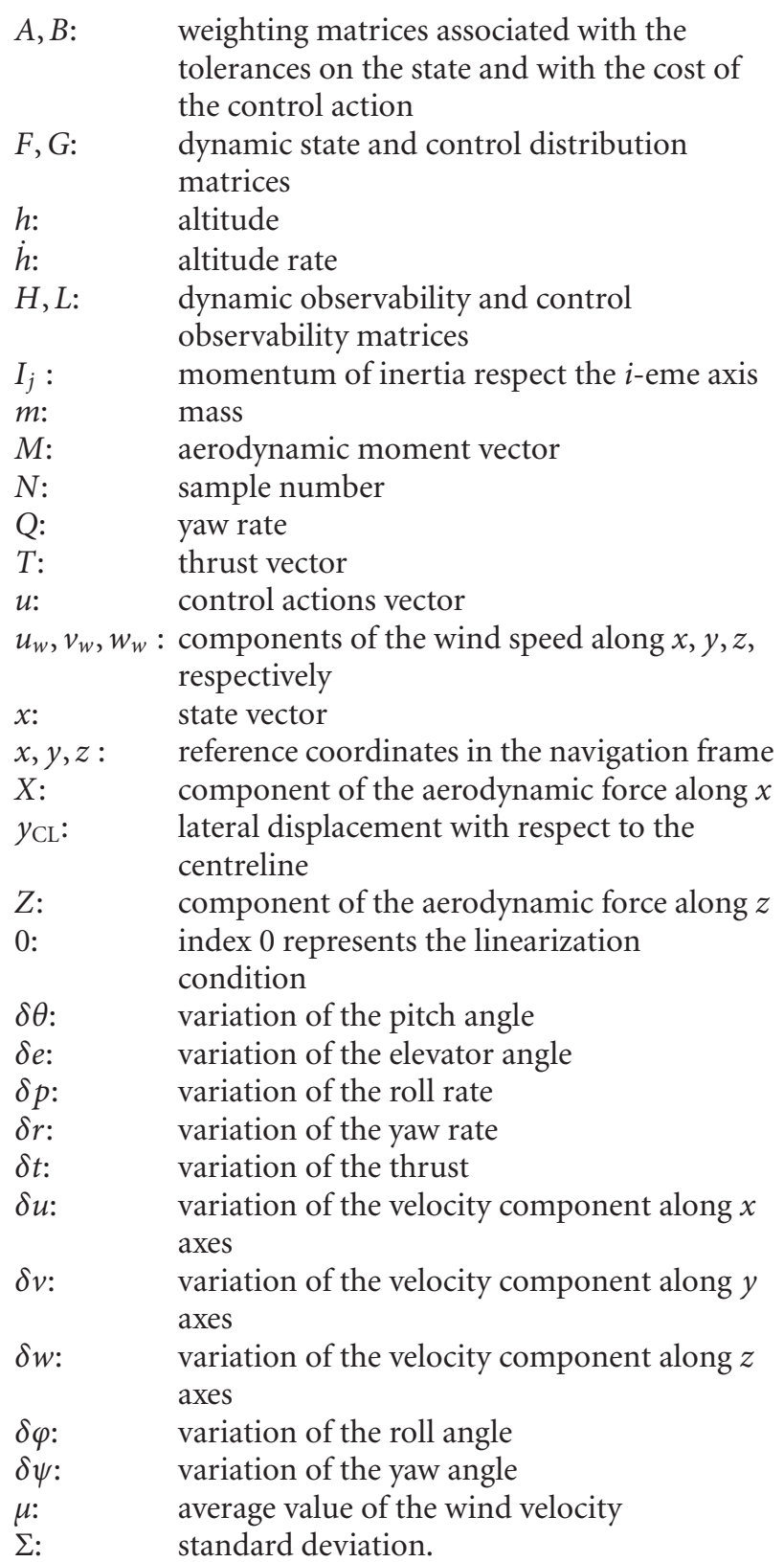

\section{Endnotes}

1. It has to be remarked that positions expressed within this work stay only with the authors. The data set recorded during November 2005 tests at the Milano Linate airport has been kindly provided to the authors and it has been only used for research applications.

\section{References}

[1] EUROCONTROL, "Long-Term forecast IFR flight movements 2008-2030," Edition 1.0, February 2008.

[2] EUROCONTROL, "Medium-Term forecast IFR flight movements 2009-2015," Edition 1.0, February 2009.

[3] ICAO DOC 9613, Performance-Based Navigation (PBN) Manual, ICAO, Quebec, Canada, 3rd edition, 2008.
[4] A. E. Bryson Jr., Control of Spacecraft and Aircraft, Princeton University Press, Englewood Cliffs, NJ, USA, 1994.

[5] R. E. Kalman, "A new approach to linear filtering and prediction problems," Journal of Basic Engineering Series D, vol. 82, pp. 35-45, 1960.

[6] R. E. Kalman and R. Bucy, "New results in linear filtering and prediction problems," Journal of Basic Engineering Series D, vol. 83, pp. 95-108, 1961.

[7] D. J. Biezad, Integrated Guidance and Navigation Systems, AIAA, Wahignton, DC, USA, 1999.

[8] R. C. Nelson, Flight Stability and Automatic Control, McGrawHill, New York, NY, USA, 1989.

[9] P. D. Tromboni, Applicazioni di navigazione satellitare alla gestione del traffico aereo, Ph.D. thesis, Università di Roma "La Sapienza", Rome, Italy, 2008.

[10] P. D. Tromboni and G. B. Palmerini, "Utilizzo del segnale EGNOS per la navigazione aerea," in Proceedings of the 19th Congresso Nazionale AIDAA, Forlì, Italy, September 2007.

[11] E. D. Kaplan and C. J. Hegart, Understanding GPS: Principles and Applications, Artech House, London, UK, 2006.

[12] B. W. Parkinson and J. Spilker Jr., Eds., Global Positioning System: Theory and Applications, vol. 1-2, AIAA, Washington, DC, USA, 1996.

[13] ICAO DOC 8168 OPS/611, Aircraft Operations-Vol. 1 Flight Procedures, 2006.

[14] C. E. Cohen, B. S. Pervan, H. S. Cobb, D. G. Lawrence, J. D. Powell, and B. W. Parkinson, "Precision landing of aircraft using integrity beacons," in Global Positioning System: Theory and Applications, B. W. Parkinson, J. J. Spilker Jr., P. Axelrad, and P. Enge, Eds., vol. 2, pp. 427-459, AIAA, Washington, DC, USA, 1996.

[15] B. W. Parkinson, M. L. O'Connor, and K. T. Fitzgibbon, "Aircraft automatic approach and landing using GPS," in Global Positioning System: Theory and Applications, B. W. Parkinson, J. J. Spilker Jr., P. Axelrad, and P. Enge, Eds., vol. 2, pp. 397-426, AIAA, Washington, DC, USA, 1996.

[16] ICAO Annex 10, Radio Navigation Aids, vol. 1, ICAO, Quebec, Canada, 5th edition, 1996. 

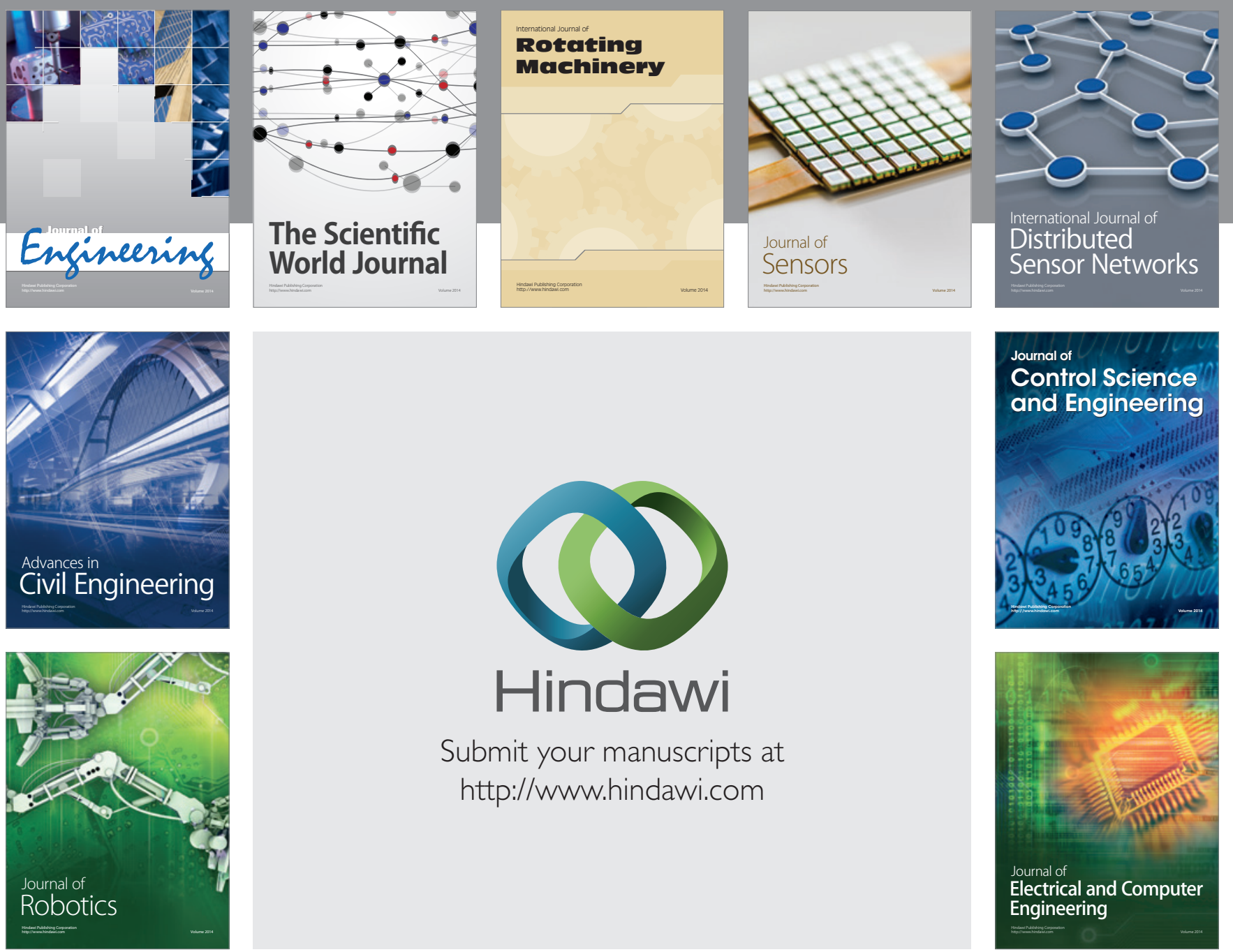

Submit your manuscripts at

http://www.hindawi.com
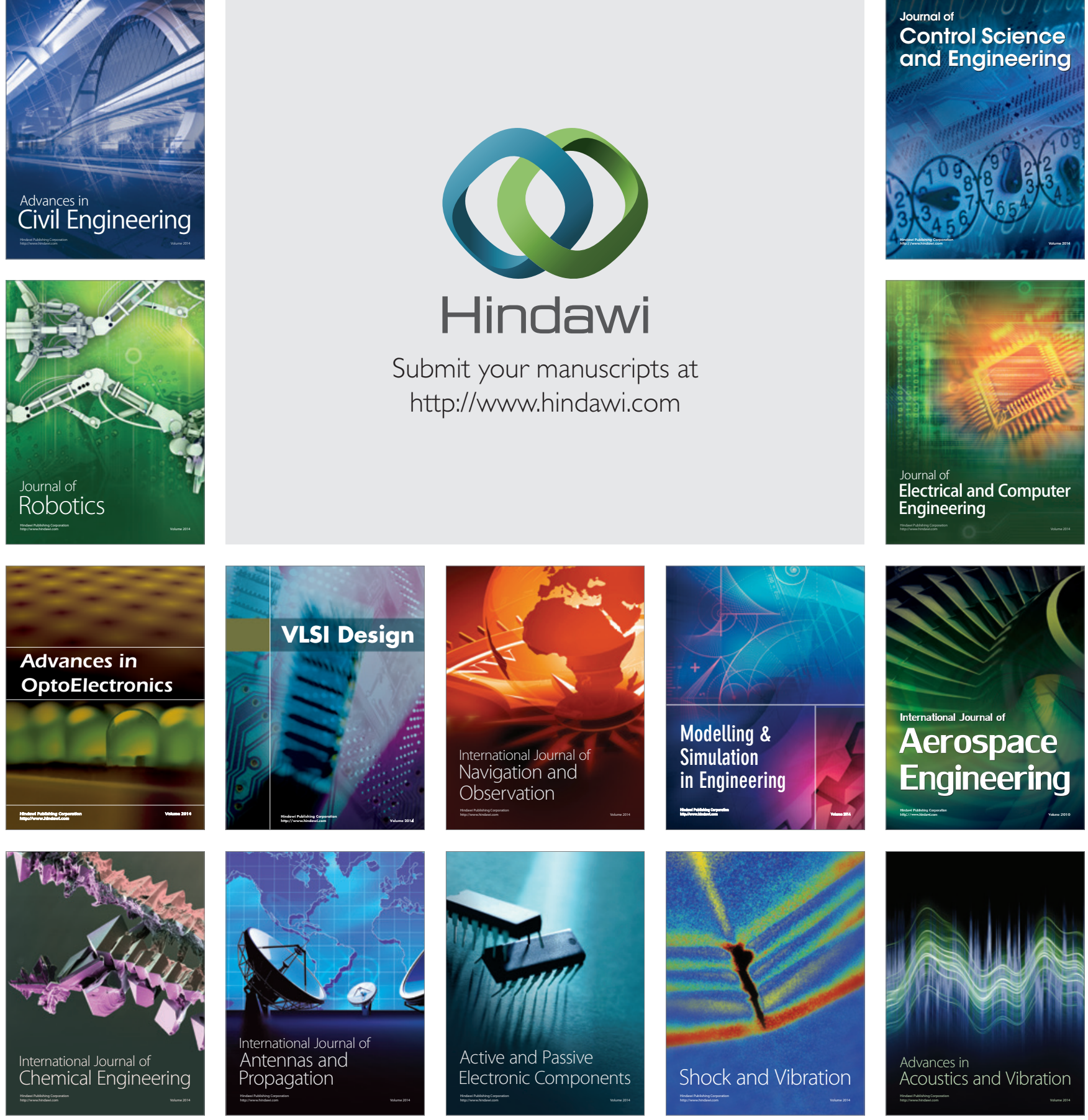